\title{
ORIGINAL ARTICLE Platelet-activating factor (PAF) receptor as a promising target for cancer cell repopulation after radiotherapy
}

\author{
IA da Silva-Jr ${ }^{1}$, R Chammas ${ }^{2}$, AP Lepique ${ }^{1}$ and S Jancar ${ }^{1}$
}

\begin{abstract}
A major drawback of radiotherapy is the accelerated growth of the surviving tumor cells. Radiotherapy generates a variety of lipids that bind to the receptor for platelet-activating factor, expressed by cells in the tumor microenvironment. In the present study, using the TC-1 tumor cell line, we found that irradiation induced a twofold increase in receptor expression and generated agonists of receptor. Irradiated cells induced a 20 -fold increase in live TC-1 proliferation in vitro. Furthermore, subcutaneous co-injection of irradiated TC-1 cells with TC-1 expressing luciferase (TC-1 fluc ${ }^{+}$) markedly increased TC-1 fluc ${ }^{+}$proliferation in a receptor-dependent way. Moreover we used a human carcinoma cell line not expressing the PAF receptor (KBM) and the same cell transfected with the receptor gene (KBP). Following co-injection of live KBP cells with irradiated KBM in RAG mice, the tumor growth was significantly increased compared with tumor formed following co-injection of live KBM with irradiated KBM. This tumor cell repopulation correlated with increased infiltration of tumor-promoting macrophages (CD206+). We propose that receptor represents a possible target for improving the efficacy of radiotherapy through inhibition of tumor repopulation.
\end{abstract}

Oncogenesis (2017) 6, e296; doi:10.1038/oncsis.2016.90; published online 30 January 2017

\section{INTRODUCTION}

Although radiotherapy is an effective way to control cancer locally, a major drawback of this treatment is the accelerated growth of surviving cells. This compensatory proliferation is an evolutionarily conserved process involved in tissue regeneration in lower animals, and is thought to occur with tumor cells treated with cytotoxic radiotherapy, as previously discussed. ${ }^{1}$ The alkyl- acyl-glycerophosphocholine (GPC), platelet-activating factor (PAF), binds to the PAF receptor. Irradiation generates reactive oxygen species, ${ }^{2}$ which act on membrane GPC to produce oxidized GPC, which also bind to the PAF receptor. ${ }^{3}$ Mass spectrometry revealed several different oxidized GPC molecules that bind to the PAF receptor. ${ }^{4,5}$ Moreover, apoptotic and necrotic cells are phagocytosed by macrophages through scavenger receptors, ${ }^{6}$ and this process involves the association of CD36 with the PAF receptor. ${ }^{7}$ These macrophages acquire an anti-inflammatory phenotype, which is thought to explain the silent nature of this process, as it does not induce inflammation. ${ }^{8}$ Tumor irradiation may induce an anti-inflammatory microenvironment that favors tumor growth. Indeed, injection of apoptotic cells with a sub-tumorigenic dose of melanoma cells was found to promote tumor growth, and this was reversed by blocking PAF receptor signaling. ${ }^{9}$ The generation of anti-inflammatory macrophages and PAF receptor agonists in the tumor microenvironment may therefore represent possible mechanisms underlying radiotherapy failure. Furthermore, some tumor cells express the PAF receptor, and activation of this receptor with PAF increased the proliferation of human SKmel-23 melanoma cells. ${ }^{10}$ In the present study, we examined the effect of gamma radiation on the proliferation of PAF receptor-expressing tumor cells, and tumor cell repopulation.

\section{RESULTS}

Irradiation increases the expression of the PAF receptor in TC-1 carcinoma cells and generates PAF receptor agonists

The murine carcinoma cell line TC- 1 was analyzed for the expression of the PAF receptor by flow cytometry analysis. The unfilled peaks represent specific antibody binding to the PAF receptor and, irradiation ( 4 and $8 \mathrm{~Gy}$ ) significantly increased PAF receptor expression (Figure 1a). PAF receptor mRNA levels also increased with irradiation (2, 4 or $8 \mathrm{~Gy})$, in a dose-dependent manner (Figure 1b). The lipid extract from the irradiated culture of TC-1 cells was then assayed for PAF receptor agonistic activity. This was achieved with an assay that uses KBM cells transfected with the PAF receptor (KBP cells), which secrete (interleukin) IL-8 in response to receptor activation. This is a very useful assay as it allows the detection of the collection of PAF receptor agonists generated by irradiation. Figure 1c shows that irradiation increased PAF receptor activation in a dose-dependent manner, compared with the non-irradiated cells. TC-1 cells irradiated with $8 \mathrm{~Gy}$ generated PAF receptor agonistic activity equivalent to $100 \mathrm{~nm}$ of PAF. Irradiation-induced dose-dependent apoptotic and necrotic cell death, shown in Figure 1d. Pre-treatment of TC-1 cells with the PAF receptor antagonist CV3988 before irradiation, significantly increased further cell death, supporting the protective effect of PAF on irradiated cells.

PAF receptor mechanisms are implicated in increased tumor cell proliferation induced by radiation

The ability of TC- 1 cells to stimulate the growth of non-irradiated TC- 1 cells was tested according to the protocol illustrated in Figure 2a. A 'feeder layer' of TC-1 cells $\left(2 \times 10^{5}\right)$ was left to rest in culture for $24 \mathrm{~h}$ before irradiation with 4 or $8 \mathrm{~Gy}$. A smaller number $\left(10^{3}\right.$ cells $)$ of TC- 1 fluc $^{+}$was then added to the cultures and a

\footnotetext{
${ }^{1}$ Departamento de Imunologia, Instituto de Ciências Biomédicas, Universidade de São Paulo, Sao Paulo, Brazil and ${ }^{2}$ Faculdade de Medicina da Universidade de São Paulo, Instituto do Câncer do Estado de São Paulo, São Paulo, Brazil. Correspondence: IA da Silva Junior, Departamento de Imunologia, Instituto de Ciências Biomédicas, Universidade de São Paulo, Av. Prof Lineu Prestes, 1374, São Paulo 05508-030, Brazil.

E-mail: iasjr@usp.br

Received 4 October 2016; revised 28 November 2016; accepted 7 December 2016
} 

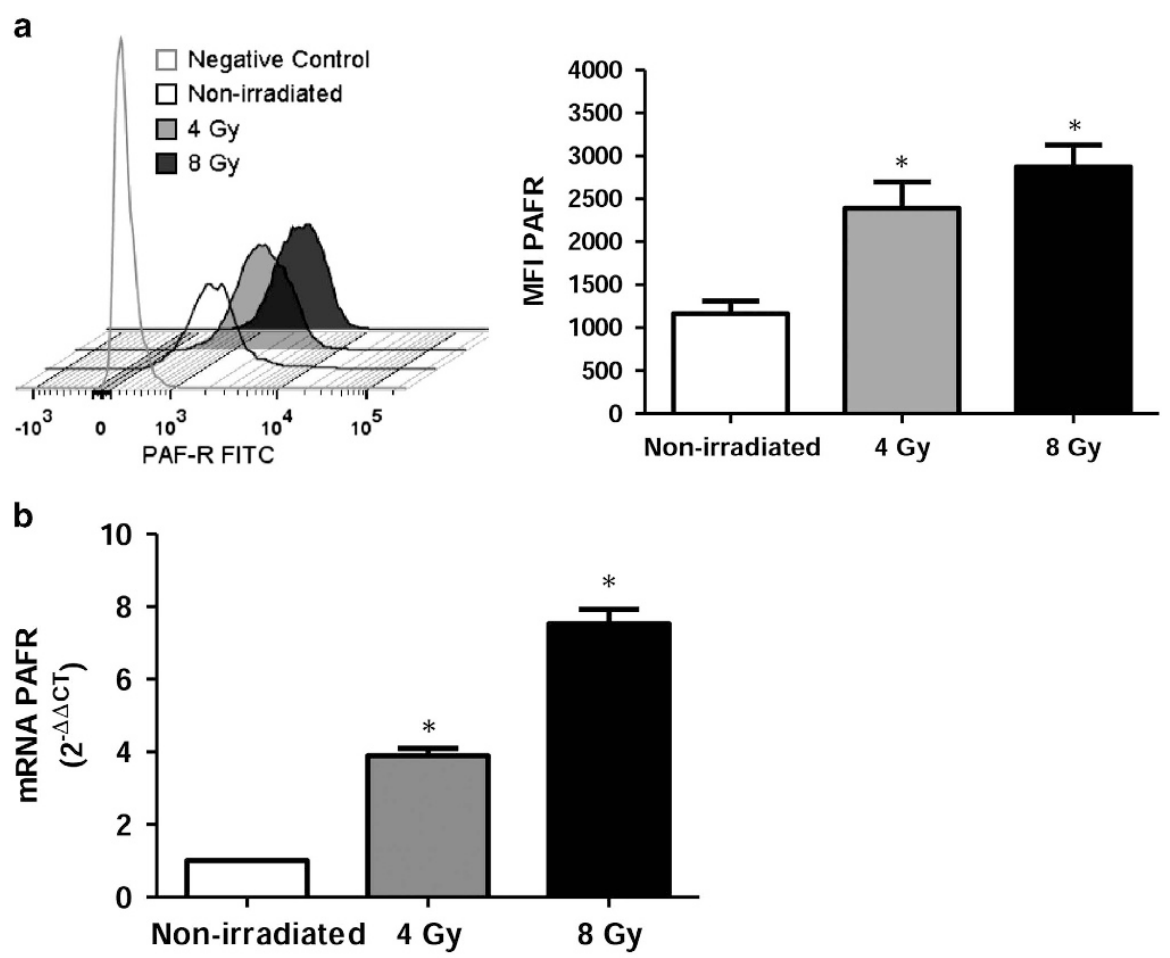

c
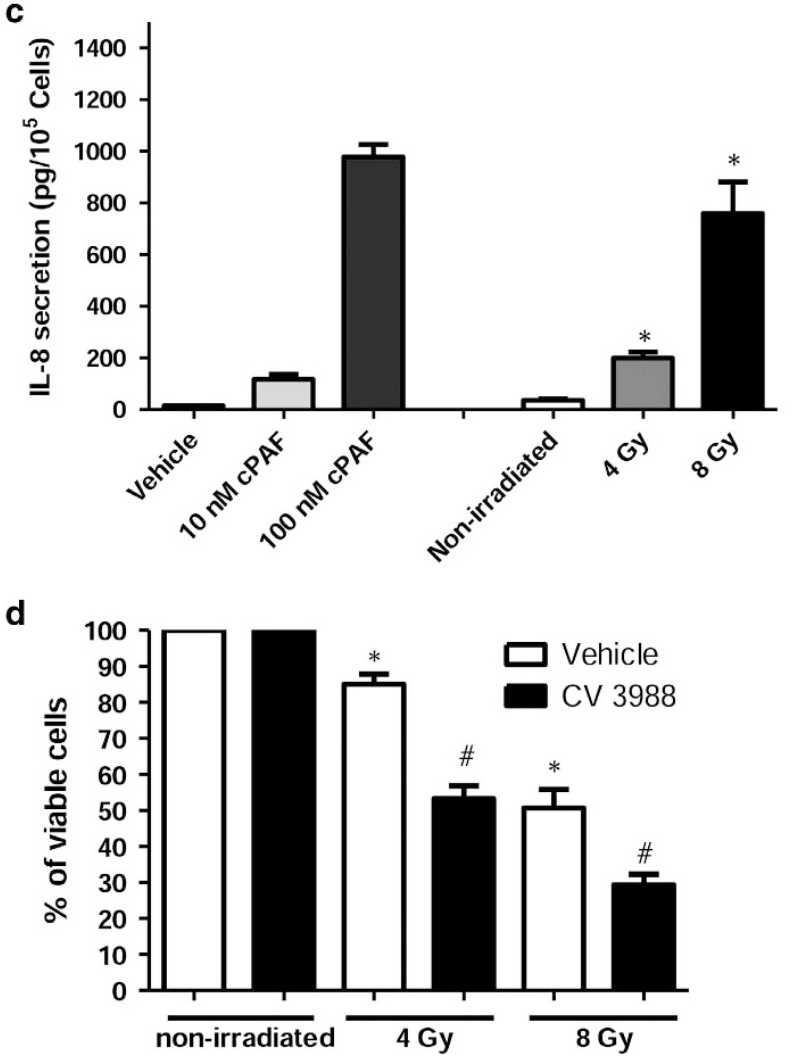

Figure 1. Radiation increases PAF receptor expression in TC- 1 cells and induces generation of PAF receptor agonists. TC- 1 tumor cells $\left(5 \times 10^{4}\right.$ ) were cultured in RPMI medium containing $2 \%$ FBS and were exposed to different doses of ionizing radiation. (a) Flow cytometry analysis of membrane PAF receptor on TC-1 cells, filled and empty traces represent non-specific and specific binding for the PAF receptor, respectively. One representative experiment out of three is shown. Mean \pm s.e.m. of MFI values ( ${ }^{*} \pm 0.05$ irradiated vs non-irradiated); (b) RNA was extracted after $4 \mathrm{~h}$ of irradiation and PAF receptor mRNA levels were measured by real-time RT-PCR and represent the mean \pm s.e.m. of six experiments made in duplicate $\left({ }^{*} P<0.05\right.$ compared to non-irradiated control); (c) The TC-1 cells culture was collected after $1 \mathrm{~h}$ of irradiation and lipids extracts were assayed for PAF receptor agonistic activity, measured as IL-8 production by PAF receptor-expressing KBP cells. As control, PAF $(10$ and $100 \mathrm{~nm})$ was added to KBP cells. Mean \pm s.e.m.* of six experiments made in duplicate $<0.005$, comparing irradiated with non-irradiated control; (d) Blocking of the PAF receptor with CV3988 potentiates irradiation-induced cell death. Mean \pm s.e.m. of four experiments made in duplicate $\left(P<0.005{ }^{*}\right.$ comparing irradiated vs non-irradiated and \#comparing CV3988 vs vehicle-treated). 
a

In vitro tumor cell repopulation model

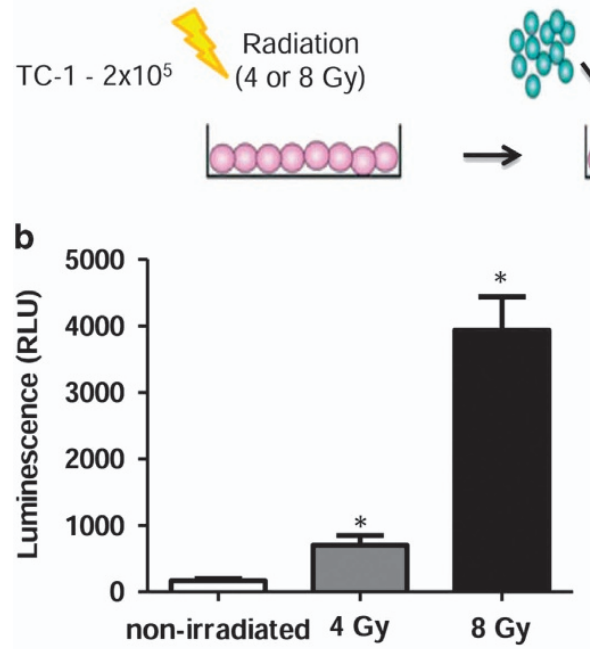

TC-1Fluc + cells $\left(10^{3}\right)$

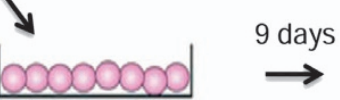

Fluc assay

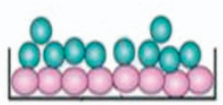

c

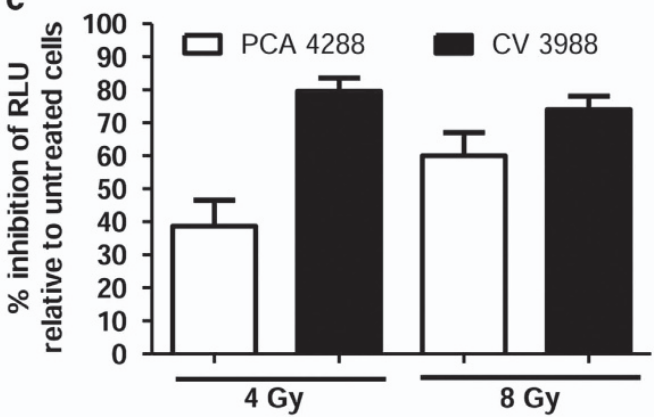

Figure 2. Irradiated cells exerts a 'feeder' effect on TC-1 cells via PAF receptor-dependent mechanism. (a) Schematic representation of experimental protocol: $2 \times 10^{5}$ TC- 1 cells were irradiated with 4 or 8 Gy and co-cultivated with $10^{3}$ TC- 1 fluc ${ }^{+}$cells for 9 days; (b) Relative luminescence units (RLU) in TC-1 cells irradiated ( 4 or $8 \mathrm{~Gy}$ ); (c) \% inhibition of RLU in cells pre-treated with PAF receptor antagonists PCA4248 or CV3988, both at $10 \mu \mathrm{m}$, relative to untreated cells. Data represent the mean \pm s.e.m. of six experiments done in duplicates. The difference between each of the irradiated groups ( 4 and $8 \mathrm{~Gy}$ ) and controls ( $0 \mathrm{~Gy})$ and between treated or not with PAF receptor antagonists was statistically significant $\left({ }^{*} P<0.05\right)$.

luciferase assay was performed following a 9 day incubation. Luminescence was linear with TC-1 fluc ${ }^{+}$cell number (Supplementary Figure 1). The presence of irradiated feeder cells significantly potentiated TC-1 proliferation, as shown in Figure $2 \mathrm{~b}$. When TC-1 fluc ${ }^{+}$cells were treated with the PAF receptor antagonists before irradiation (4 Gy), PCA4288 caused 40\% reduction in cell proliferation and CV3988 caused $80 \%$ reduction, when compared with the control, vehicle-treated cells. Following treatment with a higher dose of irradiation (8 Gy), both PAF receptor antagonists were similarly effective in their reduction of cell proliferation (Figure 2c). To evaluate whether these observations also occur in vivo, TC- 1 cells $\left(2 \times 10^{5}\right)$ were irradiated with $10 \mathrm{~Gy}$ and injected subcutaneously together with a smaller number $\left(1 \times 10^{4}\right)$ of non-irradiated TC- 1 fluc ${ }^{+}$cells, in animals with or without PAF receptor antagonist (CV3988) incubation, as illustrated in Figure 3a. As shown in Figure 3c, when live TC-1 cells were mixed with irradiated cells, the tumor volume was significantly larger than those mixed with viable cells. The treatment with the PAF receptor antagonist reduced the growth of the tumor formed by the mixture of live with irradiated cells (Figure $3 \mathrm{~d}$ ). Consistent with the in vitro observation (Figure 2 ), the irradiated cells promoted increased proliferation of the TC-1-Fluc viable cells at day 15 (Figure 3e), and the treatment with CV3988 diminished this cell proliferation and tumor size. Moreover, the lipid extracts from these tumors showed increased level of PAF receptor agonistic activity, measured as IL-8 production and subsequent activation for the PAF receptor in KBP cells (Figure 3b). Taken together, the in vitro results indicate that PAF-like molecules are produced by radiation, enhancing the proliferation of live TC-1 fluc $^{+}$cells. The in vivo results indicate that the presence of irradiated cells stimulates the growth of live tumor cells and a release of PAF receptor agonists in the tumors.

$\mathrm{PGE}_{2}$ can mediate tumor growth, induced by apoptotic cells through $\mathrm{EP}_{2}$ signaling. ${ }^{9}$ We have previously observed that the high levels of $\mathrm{PGE}_{2}$ present in Ehrlich ascites tumor were significantly reduced in mice treated with PAF receptor antagonists, suggesting that the production of $\mathrm{PGE}_{2}$ is dependent on PAF receptor ligands present in the ascites. ${ }^{11}$ Therefore, we stimulated TC-1 cells with CPAF $(100 \mu \mathrm{m})$ and found that it induced $\mathrm{PGE}_{2}$ production. Moreover, irradiation ( $8 \mathrm{~Gy}$ ) of TC-1 cells also induced $\mathrm{PGE}_{2}$ production and it was significantly reduced by the PAF receptor antagonist, CV3988 (Supplementary Figure 2).

PAF receptor and radiation-induced tumor cell repopulation To investigate if PAF receptor-mediated mechanisms are involved in tumor repopulation after radiotherapy, human carcinoma cells (KBM) that do not express PAF receptor, and were irradiated, were co-injected with the same cells transfected with the PAF receptor (KBP). In this case, cells were injected into C57BL6 Rag KO mice to avoid rejection of the human tumor cells. This model has the advantage of avoiding the interference of adaptive immunity on tumor growth, allowing the role of innate immunity on tumor repopulation to be determined. Figure 4a shows that treatment of $\mathrm{KBP}$ cells with the PAF receptor agonist CPAF (100 nM) resulted in increased in vitro proliferation, whereas the KBM cells did not proliferate in response to CPAF treatment (Figure $4 b$ ). When these cells were injected subcutaneously into RAG KO mice, both tumors grew slowly and after 30 days, KBM and KBP developed into tumors of similar volume (Figure $5 \mathrm{a}$ ). However, when the KBP was mixed with irradiated (10 Gy) KBM cells, the tumor grew rapidly and was significantly larger than the tumor that resulted from the mixture of KBM cells with irradiated KBM cells (Figure 5b).

\section{The PAF receptor and tumor macrophages}

Cells that do not survive radiotherapy are cleared from the tumor environment by tumor macrophages, in a process that is dependent on the PAF receptor. During this process, macrophages acquire the anti-inflammatory phenotype $\mathrm{M} 2{ }^{7}$ which has been shown to stimulate tumor angiogenesis and tumor growth. ${ }^{12}$ We investigated the tumor macrophages phenotype, using the KBM or KBP cells mixed with irradiated KBM cells and injected subcutaneously. After 30 days, the tumors were excised and examined for leukocytes infiltration by flow cytometry. For flow cytometry analysis, we first made a gate on the leukocyte population $(\mathrm{CD} 45+)$ and within this population analyzed the macrophage population ( $\mathrm{F} 4 / 80+)$. The latter was then analyzed using the M2 marker, CD206. A clear relationship between the 
a
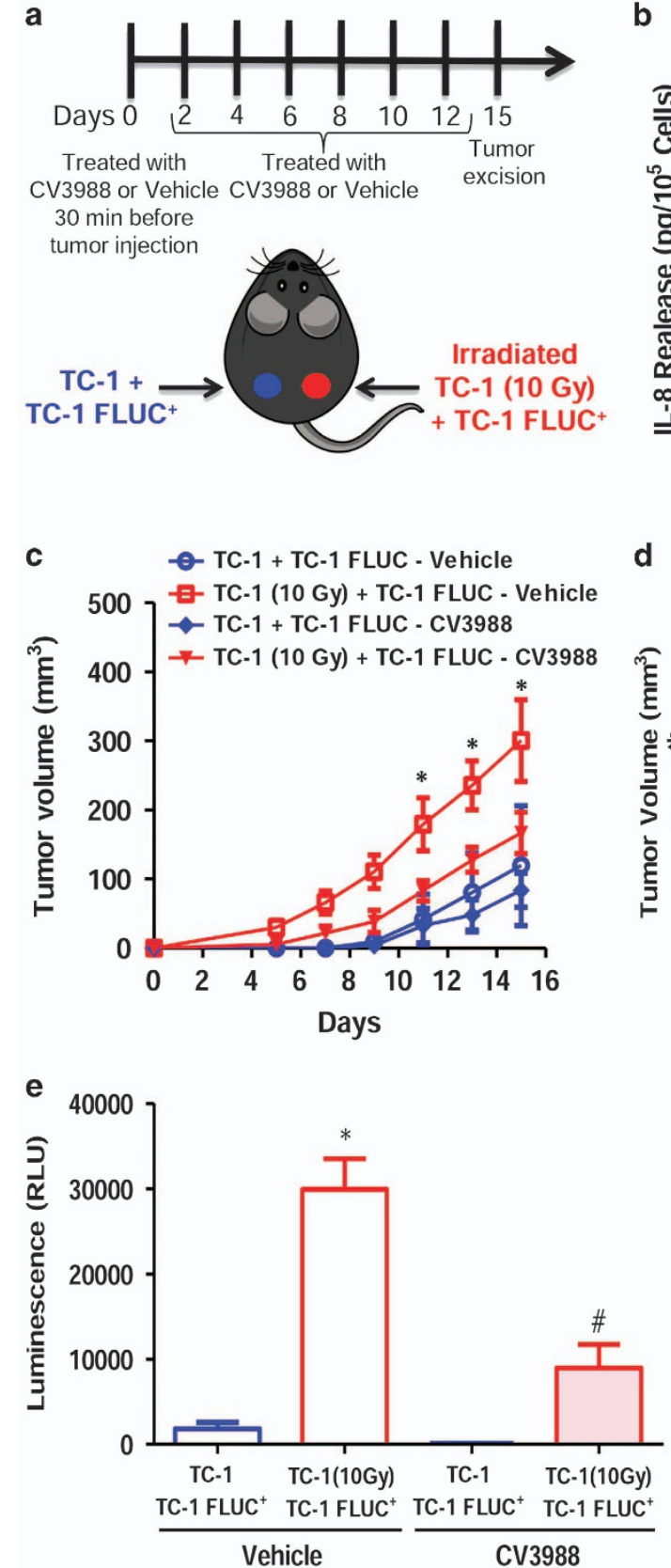

b

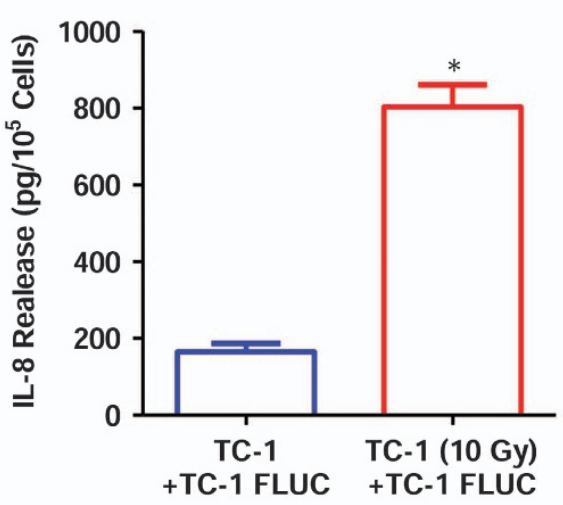

d


CV3988

Figure 3. TC-1 tumor cells growth in vivo is increased when mixed with irradiated cells. (a) Schematic representation of the protocol used in these experiments. Irradiated (10 Gy) or control TC-1 cells $\left(5 \times 10^{5}\right)$ were injected subcutaneously into the shaved back of C57BL6 mice together with non-irradiated $10^{3} \mathrm{TC}-1$-Fluc. These animals were treated with intraperitoneal injection of the PAF receptor antagonist (CV3988) in a dose of $10 \mathrm{mg} / \mathrm{kg}$ or vehicle (PBS) every 2 days; (b) PAF receptor agonistic activity in lipid extracts from tumor mass after $1 \mathrm{~h}$ of $10 \mathrm{~Gy}$, measured as IL-8 production by PAF receptor-transfected KBP cell $(n=6)$. (c) Effect of dying TC-1 on TC-1 fluc ${ }^{+}$tumor cell growth in vivo, shown as tumor volume $\left(\mathrm{mm}^{3}\right)$ followed during 15 days in the presence or absence of CV3988; (d) focus on the differences in tumor volume between groups at day 15; (e) Left, quantification of bioluminescent signals from tumors. Right, representative bioluminescent images of mice treated (right) or non-treated (left) with the PAF receptor antagonist ( $n=7$ animals per group).

presence of the PAF receptor in the KBP tumor cells and increased infiltration of leukocytes was observed (Figure 6a). Moreover, the frequency of tumor macrophages was higher with the KBP cells than the KBM (Figure 6b). In addition, the KBP tumors compared with KBM showed a higher influx of cells expressing CD206, which is a marker for tumor-promoting M2 macrophages (Figure $6 \mathrm{c}$ ).

\section{DISCUSSION}

Our results show that irradiation increased PAF receptor expression in the TC-1 tumor cell line and generated molecules that activate the PAF receptor. Irradiated cells exerted a feeder effect on live TC-1 cells in vitro by stimulating cell proliferation. Irradiated TC-1 injected subcutaneously in mice together with TC-1 fluc ${ }^{+}$, markedly increased tumor growth. In an in vivo repopulation experiment, when KBP cells were co-injected with irradiated KBM cells, the tumors were much larger than the ones formed by coinjection of KBM cells with irradiated KBM cells. The repopulation phenomenon correlated with increased frequency of M2 macrophages. A possible interpretation of these results is that irradiation induces cell death and generates molecules that bind to the PAF 
a
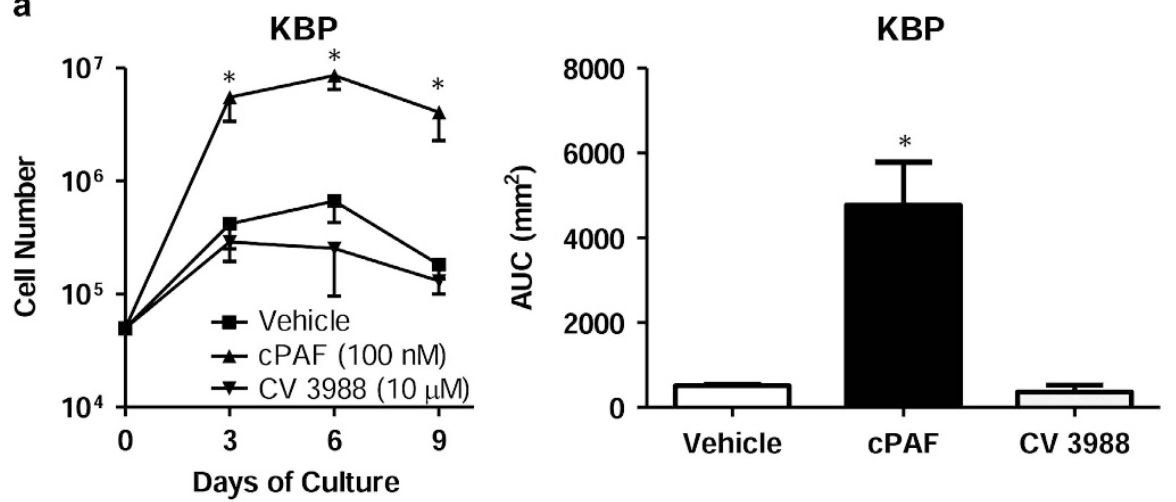

b

KBM
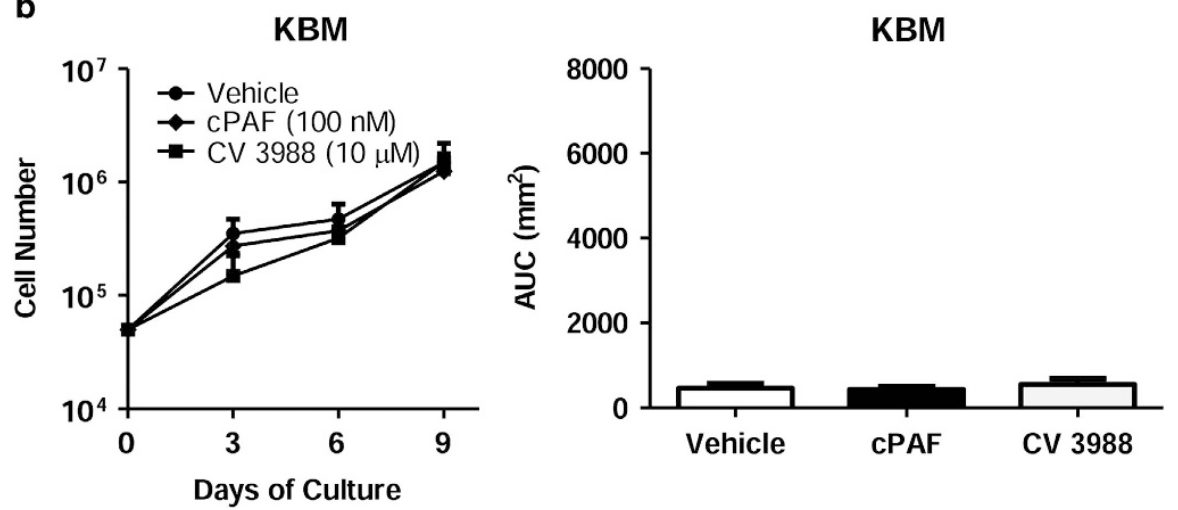

Figure 4. PAF receptor agonist (CPAF) augments tumor cell proliferation in vitro. (a) KBP cells and (b) KBM cells treated with either vehicle or CPAF $(100 \mathrm{~nm})$ or CV3988 $(10 \mu \mathrm{m})$. The cell growth was evaluated every 3 days until 9 days. The data are represented as mean \pm s.e.m. of cell number (left panel) or area under curve (right panel) from six independent experiments. Significant difference was observed between cells treated with CPAF or vehicle $(* P<0.05)$.

receptor. When injected in vivo, the irradiated cells may create a 'niche' in which the live tumor cells would have an accelerated growth, dependent on activation of the PAF receptor in tumor cells as well as tumor macrophages. Dying cells express moieties in their membranes that bind to PAF receptor ${ }^{13}$ and are cleared by macrophages by mechanisms that involve the scavenger receptor CD36 and PAF receptor. ${ }^{7}$

In the first part of this study, we show that TC-1 cells express the PAF receptor and treatment with PAF receptor agonist stimulated tumor cell proliferation. In various types of cells, PAF treatment induces phosphatidylinositol hydrolysis, arachidonic acid release and MAP kinases activation that trigger proliferative signals in tumor cells. ${ }^{14}$ Expression of the PAF receptor has been reported in pro-myelocytic leukemia cells, ${ }^{15}$ colorectal carcinoma, ${ }^{16}$ human esophageal cancer cells ${ }^{17}$ and human breast carcinoma. ${ }^{18}$ Moreover, PAF is thought to have an important role in tumor growth. ${ }^{19,20}$

The present study describes that gamma radiation of TC-1 cells generated significant levels of PAF receptor agonistic activity, assayed using a KBP cell, IL-8 releasing assay. This assay allows for detection of all ligands of the PAF receptor, and not only the agonist PAF present. Recently it was shown that chemotherapeutic agents and radiotherapy induce production of PAF-like molecules ${ }^{21,22}$ and a plethora of oxidized phospholipids that can bind to the PAF receptor. Therefore, despite cell death induced by these treatments, they also induce generation of PAF-like molecules, which may promote proliferation of treatmentresistant cells, by activating the PAF receptor expression in these cells. This study reported that irradiation induced PAF receptor ligands and promoted overexpression of the PAF receptor in tumor cells, which was found correlate with tumor cell repopulation. Blocking the PAF receptor with the antagonist CV3938 or
PCA4280 before irradiation further decreased TC-1 cell viability, suggesting that PAF receptor agonists protect tumor cells from death induced by radiotherapy. We also observed that blocking of PAF receptor abolished the effect of irradiation-induced TC-1 proliferation. To our knowledge, the role of these signaling events in TC-1 tumor repopulation has not been previously described.

Early work from REVESZ ${ }^{23}$ described that a mixture of tumor cells killed by radiation with viable cells led to increased proliferation of the latter and that this was dependent on diffusion of a growth-stimulating metabolite. Our results identify the growth-stimulating metabolite as lipid molecules that bind to the PAF receptor.

Several studies have attempted to understand the molecular mechanisms of tumor repopulation after cytotoxic therapy. Huang et al. 2011 showed that activated caspase-3 in tumor cells undergoing apoptosis has a major role in repopulation after radiotherapy by inducing $\mathrm{PGE}_{2}$ production. $\mathrm{PGE}_{2}$ has a crucial role in tumor cell proliferation and can be essential for tumor repopulation. In a previous study, we showed that the combination of chemotherapy (Dacarbazine) with the PAF receptor antagonist (WEB2170) reduced the proportion of caspase- 3 and COX-2-positive cells within the tumor. ${ }^{24}$ Thus, it is possible that 'PAF-like' molecules may potentiate tumor cell growth, either by attenuating the cytotoxic effects of radiotherapy or stimulating tumor cell proliferation.

It is interesting to note the activated caspase-3 leads to activation of CPLA2, ${ }^{25}$ which acts on phosphatidylcholine generating one molecule of arachidonic acid and lysophosphorycholine. Although arachidonic is a substrate for cyclooxygenases leading to prostanoid synthesis, upon acetylation, lysophosphorycholine converts into PAF. Therefore, the same reaction that 
a

C57BI/6 RAG KO
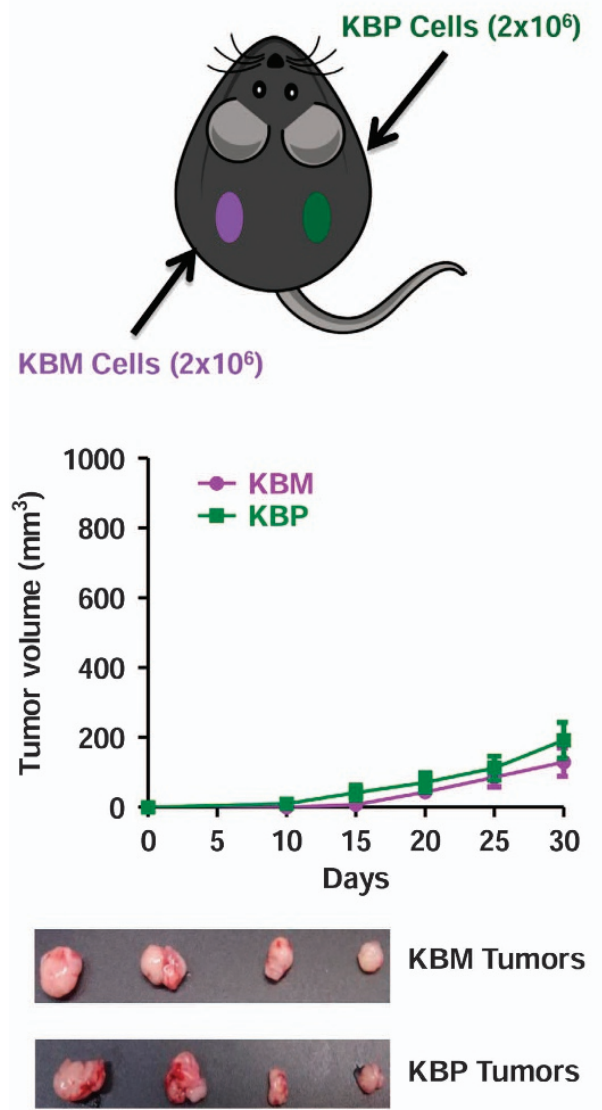

b

C57BI/6 RAG KO KBP Cells $\left(10^{6}\right)$ 10 Gy KBM Cells $\left(10^{6}\right)$

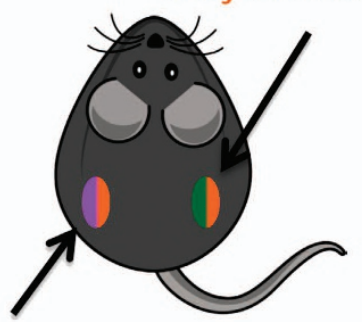

KBM Cells $\left(10^{6}\right)$

10 Gy KBM Cells $\left(10^{6}\right)$
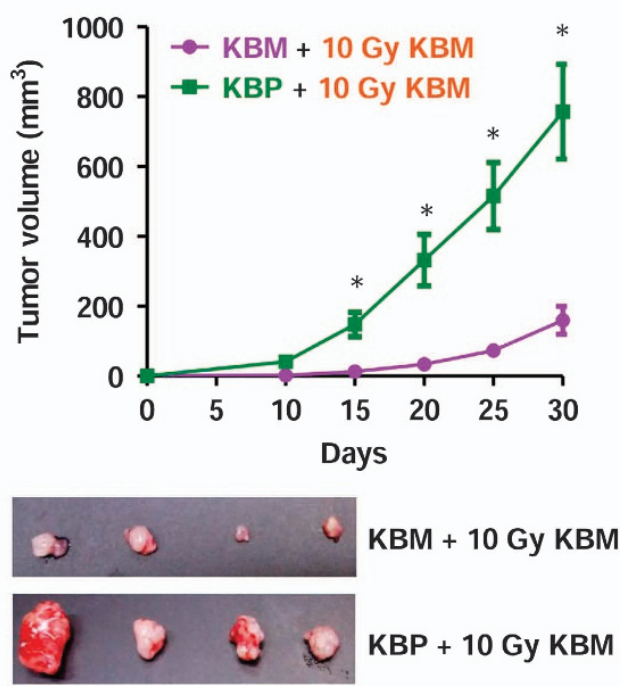

Figure 5. Repopulation is dependent on the expression of PAF receptor in the tumor cells. (a) Schematic representation of the experimental protocol. KBM or KBP cells $\left(2 \times 10^{6}\right)$ were injected subcutaneously on the dorsal hind flank skin of C57BL/6 RAG KO. (b) Mix of 1:1 live and irradiated $\mathrm{KBP} / \mathrm{KBM}$ or KBM/KBM cells in a total of $2 \times 10$ cells were injected subcutaneously in the right and left flanks of mice, respectively. *Significant differences $(P<0.05)$ were observed for KBP compared with KBM tumors. The tumors were measured with calipers every 5 days for 30 days. The data are represented as mean \pm s.e.m. of tumor volume ( $n=12$ animals per group).

stimulates PAF synthesis can stimulate $\mathrm{PGE}_{2}$ production, exerting a positive feedback loop in repopulation phenomenon.

We obtained direct evidence for a stimulatory role of PAF receptor-induced proliferation. Stimulation of KBP cells, but not of KBM cells, with carbamyl-PAF, which is not inactivated by serum acetyl-hydrolase, induced an increase in cell proliferation. These results are in accordance with the work of Bussolati et al. ${ }^{14}$ showing that endogenous or exogenous administration of PAF induces an increase in cell proliferation in breast cancer cells that express the PAF receptor without affecting proliferation in PAF receptor-negative breast cancer.

In addition, we found that injection of a large number of irradiated KBM cells mixed with KBP cells resulted in tumor growth, whereas this was not observed when KBM cells were mixed with irradiated KBM cells. This strengthens the idea that the presence of dying cells in the tumor microenvironment stimulates tumor growth.

Our results show a clear relationship between the presence of the PAF receptor in the tumor cells and increased infiltration of leukocytes, mainly composed of macrophages expressing CD206, which is a marker for tumor-promoting M2 macrophages. Although macrophages have high plasticity and can exhibit a wide range of profiles, for didactic purposes they have been grouped in defined phenotypes. ${ }^{26}$

These data suggest that PAF receptor-dependent mechanisms modify the tumor microenvironment, including the phenotype of tumor macrophages. Interactions between tumor and stromal cells can occur via cell-cell interactions, or by cytokine- or chemokine-mediated signaling. ${ }^{27}$ We propose here that these interactions also involve PAF receptor-mediated signaling favoring the tumor progression by suppressing macrophage functions.

Finally, these findings imply a dual role for the PAF receptor in tumor repopulation induced by radiotherapy. 'PAF-like' molecules generated by radiotherapy by their action on tumor cells protects them for radiation-induced cell death and by acting on macrophages, stimulates the tumor growth through immunosuppression. Therefore, association of radiotherapy with the PAF receptor antagonist represents a promising strategy for improving the efficacy of radiotherapy.

\section{MATERIAL AND METHODS}

Cell lines and culture conditions

TC-1 cells, an established murine SCC line, were kindly donated by Dr TC $\mathrm{Wu}$ (John Hopkins, Baltimore). TC- 1 fluc ${ }^{+}$cells were obtained by transfection with a vector containing the hygromycin $B$ resistance gene and the firefly luciferase gene (Fluc). Cells were maintained in Rosewell Park Memorial Institute (RPMI) medium, supplemented with $10 \%$ fetal calf serum, hygromycin B $(100 \mu \mathrm{g} / \mathrm{ml})$, neomicin $(400 \mu \mathrm{g} / \mathrm{ml})$, penicillin $(100$ units $/ \mathrm{ml})$ and streptomycin $(100 \mu \mathrm{g} / \mathrm{ml})$. In addition, we obtained a PAF receptor-negative human epithelial cell line $(\mathrm{KBM})$ and PAF receptorpositive (KBP) cells from $\mathrm{Dr}$ JB Travers (Department of Dermatology, Indiana University School of Medicine, Indianapolis, IN, USA). These cell lines were cultured in DMEM (Dulbecco's modified Eagle's medium, GIBCO, Waltham, MA, USA) supplemented with $10 \%$ fetal calf serum, penicillin 


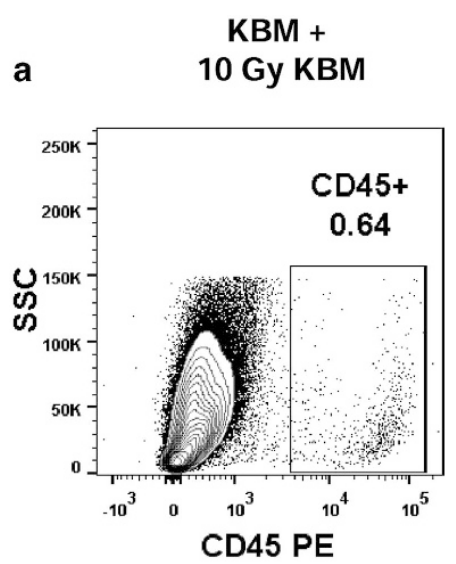

b
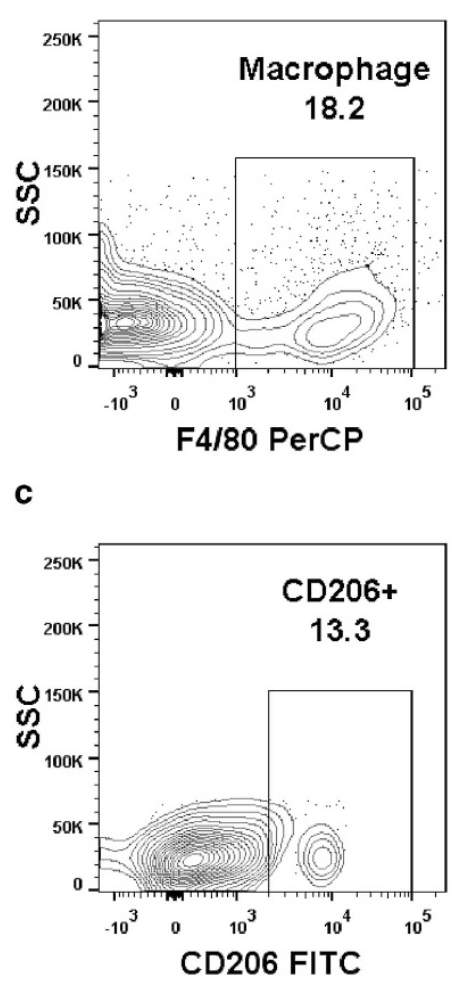

$\mathrm{KBP}+$ 10 Gy KBM
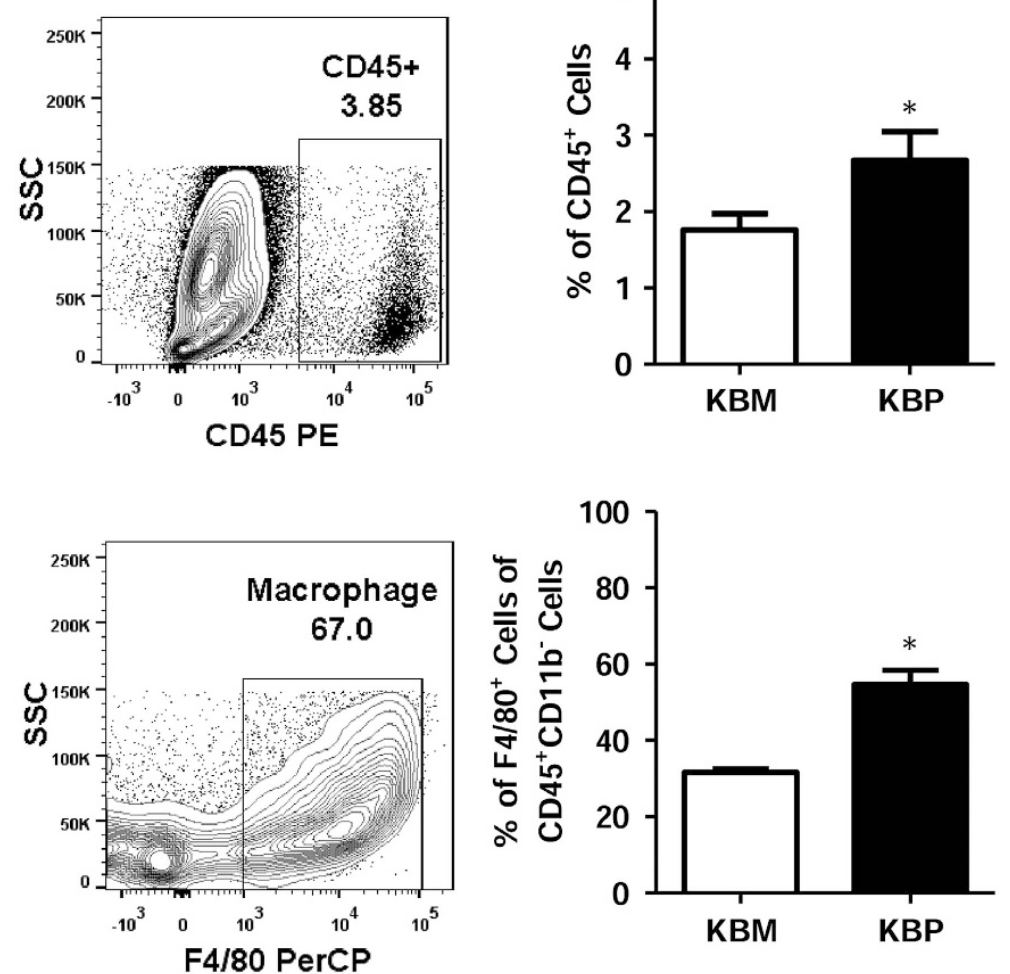

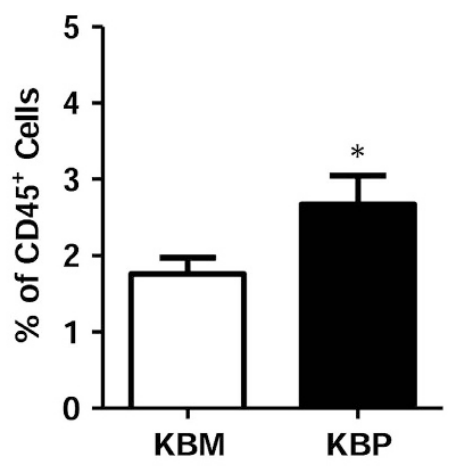

KBM

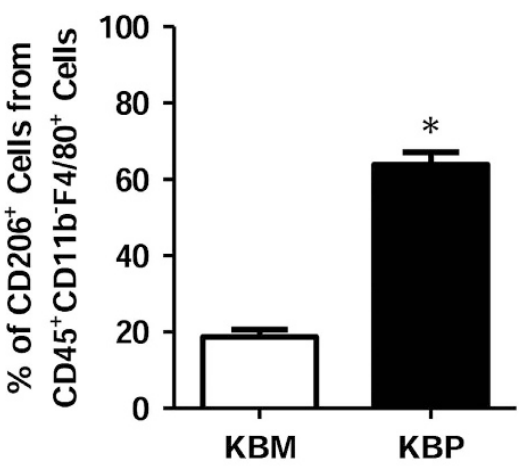

Figure 6. KBP tumor repopulation is accompanied by increased regulatory macrophages (CD206+) infiltration in the tumor mass. Macrophage analyses from tumors derived from KBM cells (left) or KBP cells (right) mixed with irradiated KBM cells (10 Gy). The tumor cells were isolated by enzymatic digestion and stained for flow cytometry analysis. Results are shown as representative plots and as means \pm s.e.m. ( $n=12$ animals per group) of (a) CD45+ population (leucocytes) (b) F4/80+ population (macrophages) and (c) CD206+ population of F4/80+ cells (M2 macrophages). ${ }^{*} P<0.05$ for KBM compared with KBP tumors.

(100 units $/ \mathrm{ml})$ and streptomycin $(100 \mu \mathrm{g} / \mathrm{ml})$. Cells have been regularly tested for Mycoplasma and were free of this contamination. All cell cultures were incubated at $37{ }^{\circ} \mathrm{C}$ under a humidified atmosphere of air containing $5 \% \mathrm{CO}_{2}$. A growth curve of control and PAF receptor agonist treated (100 nM CPAF) KBM and KBP cells was performed.

\section{Animal model}

The experiments were performed using 6- to 8-week-old male C57BL6/C or C57BL6/C RAG knockout mice from the animal breeding facility in the Department of Immunology, São Paulo University. The animal facility and subsequent experiments were approved according to the Brazilian Animal Welfare Regulations (National Council for Animal Experimentation Control - CONCEA, protocol number 130/2015). The animal rooms provided daylight plus a $12 \mathrm{~h}$ light-dark electric cycle and a constant temperature of $26{ }^{\circ} \mathrm{C}$ and relative humidity of $50-60 \%$. The mice were fed a laboratory animal diet and sterile water ad libitum. Tumor cells were injected subcutaneously as single-cell suspensions in phosphate-buffered saline (PBS; $2 \times 10^{6}$ cells in $100 \mu \mathrm{l}$ ) into the right and left hind leg of the mice. Some tumor cells were irradiated before injection, and these treatments are indicated in the appropriate Figures as well as the number of animals per group. Tumor volume was calculated using the equation: $v=\left(d_{1} \times d_{2}\right)^{2} \times 0.6$, where $V$ is the tumor volume, $d_{1}$ is the largest diameter of the tumor and $d_{2}$ is the smallest diameter of the tumor. None of the animals injected with tumor cells was excluded from the analysis. A method of randomization was not used to allocate the experimental groups. Allocation of the animals and assessment of the outcome were done without blinding.

In vitro irradiation of TC- 1 tumor cells

TC-1 cell lines were grown on $10 \mathrm{~cm}$ dishes to $80-90 \%$ confluence, and washed three times with pre-warmed $\left(37^{\circ} \mathrm{C}\right)$ PBS and then cultured in RPMI medium containing $2 \%$ fetal bovine serum. Tumor cells were 
irradiated with multiple doses of gamma radiation (Gy) and incubated for $1 \mathrm{~h}$ or $6 \mathrm{~h}$ time points, as detailed in the Figure legends. Cell irradiation studies were conducted using an IBL 136 cell and animal gamma radiator machine (Compagnie Oris Industrie, Gif-sur-Yvette, France). Settings for the machine were as follows: $d=33 \mathrm{~cm}$, and dose rate of $251.7 \mathrm{cGy} / \mathrm{min}$. In some experiments, the PAF receptor antagonist (CV3988; Enzo Life Sciences, Farmingdale, NY, USA) or the vehicle control dimethyl sulfoxide $(0.5 \%)$ were pre-incubated for 30 min before irradiation. After $48 \mathrm{~h}$, the cells were collected and counted, and cell viability examined by the trypan blue exclusion test. The expression of the PAF receptor in carcinoma tumor cell lines was assessed by flow cytometry and real-time reverse transcriptase PCR.

\section{Flow cytometry for PAF receptor expression}

Irradiated TC-1 cells and non-irradiated TC-1 cells were incubated for $48 \mathrm{~h}$. The cell culture medium was then replaced with ice-cold PBS and the cells were removed with a rubber policeman cell scraper and harvested by centrifugation at $250 \mathrm{~g}$ for $5 \mathrm{~min}$. Following centrifugation, the cell pellet was washed and re-suspended in staining buffer (PBS, fetal calf serum $1 \%$, sodium azide $0.1 \%$ ), containing the anti-PAF receptor primary antibody (1:100 dilution in staining buffer; Cayman Chemical, Ann Arbor, MI, USA). Following a $30 \mathrm{~min}$ incubation, the cells were washed and re-suspended in staining buffer containing Alexa Fluor 647-goat anti-rabbit IgG secondary antibody (1:100 dilution in staining buffer; Invitrogen-Life Technologies, Carlsbad, CA, USA). Cells incubated with secondary antibody only were used to control for background fluorescence. The expression of the PAF receptor was analyzed by flow cytometry using BDFACS-Canto II (BD Biosciences, San Jose, CA, USA) and FlowJo Version 5.0 software (TreeStar, Ashland, OR, USA). During data acquisition, the doublets were excluded using gates in FSC-A vs FSC-H. The autofluorescence of TC-1 cells was removed at the beginning of the analysis.

\section{RNA analysis for PAF receptor expression}

PAF receptor expression was analyzed by reverse transcriptase PCR using RNA obtained from TC-1 cells, collected $6 \mathrm{~h}$ post irradiation. Total RNA was isolated using TRIzol (Life Technologies). For real-time reverse transcriptase PCR, CDNA was synthesized using the RevertAid First Strand CDNA Synthesis Kit (Fermentas Life Sciences, Ontario, CA, USA), according to the manufacturer's instructions. PCR-master mix (Power SyBr Green, Applied Biosystems, Warrington, UK) containing the specific primers was then added. Human PAF receptor forward primer: GGG GAC CCC CAT CTG CCTCA and reverse GCG GGC AAA GAC CCA CAG CA; GAPDH forward primer: GAG TCA ACG GAT TTG GTC GT and reverse primer: TTG ATT TTG GAG GGA TCT CG. Real-time PCR was performed using the Mx3005PTM Real-Time PCR System. Relative gene expression was calculated using the $2{ }_{T}^{-\Delta \Delta C}$ method, as previously described. ${ }^{28}$ Results are presented as a fold increase relative to non-irradiated cells.

\section{Measurement of PAF receptor ligands after irradiation}

TC-1 cells $\left(10^{7} \mathrm{ml}\right)$ were plated in $10 \mathrm{~cm}$ dishes and incubated overnight in DMEM supplemented with $10 \%$ fetal calf serum medium. Following incubation, the culture medium was replaced with $2 \mathrm{ml}$ of pre-warmed $\left(37^{\circ} \mathrm{C}\right)$ Hanks Balanced Salt Solution, supplemented with $10 \mathrm{mg} / \mathrm{ml}$ fatty acid-free bovine serum albumin and $2 \mu \mathrm{m}$ Pefabloc, a serine hydrolase inhibitor (Sigma-Aldrich, St Louis, MO, USA). The cells were then irradiated with 2-8Gy following a $1 \mathrm{~h}$ incubation, the culture was quenched by addition of $2 \mathrm{ml}$ of ice-cold methanol followed by methylene chloride, and lipids were extracted, as previously described. ${ }^{29-31}$ The ability of irradiation to produce PAF receptor agonists was also tested in vivo, using TC-1 tumors that were implanted subcutaneously into the left hind flanks of C57BL6/c animals. Mice were anesthetized $1 \mathrm{~h}$ after irradiation and tumors dissected, weighed and lipids were extracted. The presence of PAF receptor ligands in lipid extracts was determined by the ability of these extracts to induce IL-8 production in KBP cells, as previously described. $^{32}$ In brief, $2 \times 10^{-5} \mathrm{KBP}$ cells were plated in 12 -well plates and cultured overnight in DMEM containing $10 \%(\mathrm{v} / \mathrm{v})$ fetal bovine serum. The cells were then washed with PBS, and incubated with fetal bovine serum-free DMEM. The cells were stimulated with the lipid extracts from irradiated TC- 1 cells and after $6 \mathrm{~h}$, the supernatants were collected for IL-8 measurement by ELISA. The concentration of IL-8 induced by the lipid extracts was compared with that induced by the stable PAF receptor agonist CPAF (1-hexadecyl-2-N-methylcarbamoyl glycerolphosphocholine). The concentration of IL-8 in the supernatants was measured using BD OptEIA ELISA sets (BD Biosciences, San Diego, CA, USA), according to the manufacturer's instructions.

\section{Bioluminescence imaging}

The TC-1 fluc ${ }^{+}$cells were imaged in the IVIS200 instrument (Caliper Life Sciences, Hopkinton, MA, USA). To monitor growth of fluc ${ }^{+}$-labeled cells in vitro, $\sim 1000$ cells were mixed together with $2 \times 10^{5}$ unlabeled cells, immediately after irradiation. In some experiments, blockage of the PAF receptor was performed using the chemically unrelated antagonists CV3988 or PCA4288 (Tocris Biosciences, Ellisville, MO, USA), which were added to TC-1 culture 30 min prior to irradiation. The growth of TC-1 fluc ${ }^{+}$ cells was monitored following 9 days of culture, by adding Rosewell Park Memorial Institute medium with $0.15 \mathrm{mg} / \mathrm{ml}$ D-luciferin (Caliper Life Sciences). Cells were imaged $2 \mathrm{~min}$ after the addition of D-luciferin. The growth of fluc ${ }^{+}$-labeled tumor cells in vivo was monitored by non-invasive bioluminescence imaging. Mice were imaged following intraperitoneal injection with $150 \mathrm{mg} / \mathrm{kg}$ of D-luciferin in $200 \mu \mathrm{l}$ of PBS, and then anesthetized with a continuous flow of isofluorane. Imaging of the mice was carried out 10 min later. Acquired images were analyzed following the manufacturer's instructions.

Flow cytometry analyses of macrophages from KBM/KBP tumors For FACS analyses, tumors were harvested following animal euthanasia, and digested with $100 \mu \mathrm{g} / \mathrm{ml}$ collagenase with constant stirring at $37^{\circ} \mathrm{C}$ for 45 min. Cell pellets were re-suspended in FACS buffer (PBS containing $0.1 \%$ sodium azide and $1 \%$ fetal bovine serum) and stained with fluorescentconjugated monoclonal antibodies: Pacific Blue anti-mouse CD45 Antibody (30-F11); PE-Cy5.5 conjugated F4/80 (clone BM8); FITC anti-mouse CD206 (C068C2), all purchased from eBiosciences (San Diego, CA, USA). The cell suspension was filtered through a $70 \mu \mathrm{m}$ mesh and washed twice prior to analyses by flow cytometry, as described above. Results were collected for 100000 cells and analyzed using the FlowJo software (TreeStar). F4/80+ populations were determined on CD45+ gated cells.

\section{ELISA measurement of $\mathrm{PGE}_{2}$}

To measure $\mathrm{PGE}_{2}$ secretion by TC- 1 cells, $10^{6}$ cells/well were plated in $10 \mathrm{~cm}$ dishes and incubated overnight. The cells were treated with the COX inhibitor, indomethacin (Sigma-Aldrich) or the PAF receptor antagonist (CV3988), for $30 \mathrm{~min}$ prior gamma radiation treatment. The supernatants from the cells were collected $1 \mathrm{~h}$ after cell irradiation and $\mathrm{PGE}_{2}$ levels in the supernatants were measured by ELISA (Cayman Chemical, Minneapolis, MN, USA).

\section{Statistical analysis}

Data are represented as mean \pm s.d. unless otherwise indicated and were analyzed using the Prism 5.0 statistical program (GraphPad Software, San Diego, CA, USA). No sample or animal exclusion criterion was used. Comparisons among groups were performed by analysis of variance followed by the Bonferroni multiple comparison test. A two-sided $P<0.05$ was considered statistically significant. Each experiment was repeated at least three times.

\section{CONFLICT OF INTEREST}

The authors declare no conflict of interest.

\section{ACKNOWLEDGEMENTS}

We would like to sincerely thank Marlise BA Montes for the expert technical support. This work was supported by Fundação de Amparo a Pesquisa do Estado de São Paulo (FAPESP) and Conselho Nacional de Desenvolvimento Científico e Tecnológico (CNPq).

\section{REFERENCES}

1 Zimmerman Ma, Huang Q, Li F, Liu X, Li CY. Cell Death-Stimulated Cell Proliferation: a tissue regeneration mechanism usurped by tumors during radiotherapy. Semin Radiat Oncol 2013; 23: 288-295.

2 Yamamori T, Yasui H, Yamazumi M, Wada Y, Nakamura Y, Nakamura H et al. lonizing radiation induces mitochondrial reactive oxygen species production accompanied by upregulation of mitochondrial electron transport chain function 
and mitochondrial content under control of the cell cycle checkpoint. Free Radic Biol Med 2012; 53: 260-270.

3 Patel KD, Zimmerman GA, Prescott SM, McIntyre TM. Novel leukocyte agonists are released by endothelial cells exposed to peroxide. J Biol Chem 1992; 267: 15168-15175.

4 Greenberg ME, Li X-M, Gugiu BG, Gu X, Qin J, Salomon RG et al. The lipid whisker model of the structure of oxidized cell membranes. J Biol Chem 2008; 283: 2385-2396.

5 McIntyre TM. Bioactive oxidatively truncated phospholipids in inflammation and apoptosis: formation, targets, and inactivation. Biochim Biophys Acta 2012; 1818: 2456-2464.

6 Fadok VA, Bratton DL, Henson PM. Phagocyte receptors for apoptotic cells: recognition, uptake, and consequences. J Clin Invest 2001; 108: 957-962.

7 Ferracini M, Rios FJO, Pecenin M, Jancar S. Clearance of apoptotic cells by macrophages induces regulatory phenotype and involves stimulation of CD36 and platelet-activating factor receptor. Mediators Inflamm 2013; 2013: 950273.

8 Chazaud B. Macrophages: supportive cells for tissue repair and regeneration. Immunobiology 2014; 219: 172-178.

9 Bachi ALL, Dos Santos LC, Nonogaki S, Jancar S, Jasiulionis MG. Apoptotic cells contribute to melanoma progression and this effect is partially mediated by the platelet-activating factor receptor. Mediators Inflamm 2012; 2012: 610371.

10 Onuchic AC, MacHado CML, Saito RF, Rios FJ, Jancar S, Chammas R. Expression of PAFR as part of a prosurvival response to chemotherapy: A novel target for combination therapy in melanoma. Mediators Inflamm 2012; 2012: 10-15.

11 Fecchio $D$, Sirois $P$, Russo M, Jancar S. Studies on inflammatory response induced by Ehrlich tumor in mice peritoneal cavity. Inflammation 1990; 14: 125-132.

12 Hao NB, Lü MH, Fan YH, Cao YL, Zhang ZR, Yang SM. Macrophages in tumor microenvironments and the progression of tumors. Clin Dev Immunol 2012; 2012: 948098.

13 de Oliveira SI, Fernandes PD, Amarante Mendes JGP, Jancar S. Phagocytosis of apoptotic and necrotic thymocytes is inhibited by PAF-receptor antagonists and affects LPS-induced COX-2 expression in murine macrophages. Prostaglandins Other Lipid Mediat 2006; 80: 62-73.

14 Bussolati B, Biancone L, Cassoni P, Russo S, Rola-Pleszczynski M, Montrucchio G et al. PAF produced by human breast cancer cells promotes migration and proliferation of tumor cells and neo-angiogenesis. Am J Pathol 2000; 157: 1713-1725.

15 Laurenzana A, Cellai C, Vannucchi AM, Pancrazzi A, Romanelli MN, Paoletti F. WEB-2086 and WEB-2170 trigger apoptosis in both ATRA-sensitive and -resistant promyelocytic leukemia cells and greatly enhance ATRA differentiation potential. Leuk Off J Leuk Soc Am Leuk Res Fund, UK 2005; 19: 390-395.

16 Denizot Y, Gainant A, Guglielmi L, Bouvier S, Cubertafond P, Mathonnet M. Tissue concentrations of platelet-activating factor in colorectal carcinoma: inverse relationships with Dukes' stage of patients. Oncogene 2003; 22: 7222-7224.

17 Wang LS, Chow KC, Wu YC. Effects of platelet activating factor, butyrate and interleukin- 6 on cyclooxygenase-2 expression in human esophageal cancer cells. Scand J Gastroenterol 2002; 37: 467-475.

18 Pitton C, Lanson M, Besson P, Fetissoff F, Lansac J, Benveniste J et al. Presence of PAF-acether in human breast carcinoma: relation to axillary lymph node metastasis. J Natl Cancer Inst 1989; 81: 1298-1302.

19 Fragel-Madeira L, Meletti T, Mariante RM, Monteiro RQ, Einicker-Lamas $M$, Bernardo RR et al. Platelet activating factor blocks interkinetic nuclear migration in retinal progenitors through an arrest of the cell cycle at the S/G2 transition. PLOS ONE 2011; 6: e16058.

20 Patrick C, Konger L, Jeffrey B. Systemic platelet-activating factor receptor activation augments experimental lung tumor growth and metastasis. Cancer Growth Metastasis 2014; 2: 27-32.

21 Sahu R. Expression of the platelet-activating factor receptor enhances benzyl isothiocyanate-induced apoptosis in murine and human melanoma cells. Mol Med Rep 2015; 12: 394-400.

22 Sahu RP, Harrison KA, Weyerbacher J, Murphy RC, Konger RL, Garrett JE et al. Radiation therapy generates platelet-activating factor agonists. Oncotarget 2016; 7: $20788-20800$.

23 REVESZ L. Effect of tumour cells killed by x-rays upon the growth of admixed viable cells. Nature 1956; 178: 1391-1392.

24 de Oliveira SI, Andrade LNS, Onuchic AC, Nonogaki S, Fernandes PD, Pinheiro MC et al. Platelet-activating factor receptor (PAF-R)-dependent pathways control tumour growth and tumour response to chemotherapy. BMC Cancer 2010; 10: 200.

25 Huang Q, Li F, Liu X, Li W, Shi W, Liu F-F et al. Caspase 3-mediated stimulation of tumor cell repopulation during cancer radiotherapy. Nat Med 2011; 17: 860-866.

26 Biswas SK, Mantovani A. Macrophage plasticity and interaction with lymphocyte subsets: cancer as a paradigm. Nat Immunol 2010; 11: 889-896.

27 Mezawa Y, Orimo A. The roles of tumor- and metastasis-promoting carcinoma-associated fibroblasts in human carcinomas. Cell Tissue Res 2016; 365: 675-689.

28 Livak KJ, Schmittgen TD. Analysis of relative gene expression data using real-time quantitative PCR and the 2(-Delta Delta C(T)) Method. Methods 2001; 25: 402-408.

29 Yao Y, Wolverton JE, Zhang Q, Marathe GK, Al-Hassani M, Konger RL et al. Ultraviolet $B$ radiation generated platelet-activating factor receptor agonist formation involves EGF-R-mediated reactive oxygen species. J Immunol 2009; 182: 2842-2848.

30 Sahu RP, Petrache I, Van Demark MJ, Rashid BM, Ocana JA, Tang Y et al. Cigarette smoke exposure inhibits contact hypersensitivity via the generation of platelet-activating factor agonists. J Immunol 2013; 190: 2447-2454.

31 Marathe GK, Johnson C, Billings SD, Southall MD, Pei Y, Spandau D et al. Ultraviolet $B$ radiation generates platelet-activating factor-like phospholipids underlying cutaneous damage. J Biol Chem 2005; 280: 35448-35457.

32 Ferracini M, Sahu RP, Harrison KA, Waeiss RA, Murphy RC, Jancar S et al. Topical photodynamic therapy induces systemic immunosuppression via generation of platelet-activating factor receptor ligands. J Invest Dermatol 2015; 135: 321-323.

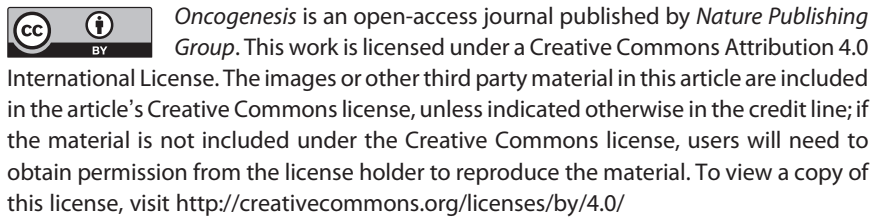

(c) The Author(s) 2017

Supplementary Information accompanies this paper on the Oncogenesis website (http://www.nature.com/oncsis) 